\title{
THE REGULARIZATION OF NONLINEAR ELECTRICAL CIRCUITS
}

\author{
E. IHRIG
}

ABSTRACT. Recently Smale [1] proposed the question 'under what conditions can an electrical circuit be regularized?' This note gives a solution to the problem.

1. Introduction. Recently Smale formulated the system of differential equations that govern an electrical circuit in a geometric fashion on the manifold of states. We refer the reader to Smale [1] for the detailed description of the terms used here and the mathematical theory.

In summary, a state manifold is defined and called $\Sigma$. A smooth map, $\pi$, is defined, $\pi: \Sigma \rightarrow \mathfrak{L} \times \mathcal{C}^{\prime}$, where $\mathfrak{L}$ is the vector space generated by the inductance branches of the circuit and $\mathcal{C}^{\prime}$ the vector space generated by the capacitance branches. The dynamical equations for the circuit turn out to be:

$$
l(d x / d t, Y)=\omega(Y) \text { for all } Y \in T(\Sigma)
$$

where $\omega$ is a given one form on $\Sigma, x(t)$ is the state of the circuit at time $t$, and $I$ is a symmetric bilinear form with $I=\pi^{*} J . J$ is a symmetric nondegenerate bilinear form on $\mathcal{L}_{\times} \mathcal{C}^{\prime}$ which is given. This equation determines $d x / d t$ and thus $x(t)$ if and only if $I$ is nondegenerate. Since $\operatorname{dim} \Sigma=$ $\operatorname{dim} \mathcal{Q} \times \mathcal{C}^{\prime}$ this condition is equivalent to $\pi$ being a local diffeomorphism.

In fact it is found that circuits for which $\pi$ is not a local diffeomorphism exist. However, one finds that by adding some small inductances and capacitances (which would exist anyway in any physical circuit) in these examples, one can make $\pi$ into a diffeomorphism. Smale poses the problem whether this can be done for a general circuit and, if so, how can it be done. It is the purpose of this note to give an answer to this problem.

2. The regularization theorem. We refer the reader to Smale [1] for our notation. We start with a definition.

\subsection{Definition.}

(i) A circuit is called regular if $\pi$ is a diffeomorphism $\pi: \Sigma \rightarrow \mathscr{Q} \times \mathfrak{C}^{\prime}$.

(ii) A circuit is called regularizable if a new regular circuit can be 
obtained from the original circuit by the addition of a number of small inductors and capacitors.

\subsection{Example.}

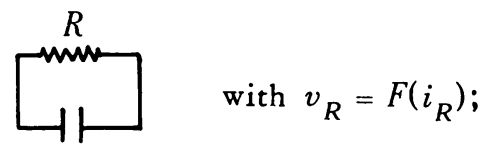

$F$ is some smooth function. This circuit is regularized by

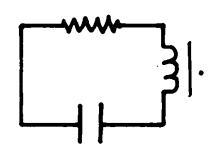

We now proceed with our main result.

2.3. Theorem. Suppose we are given a circuit $\Gamma$ in which, for each resistance branch $\rho$, we have either (a) $v_{\rho}=F_{\rho}\left(i_{\rho}\right)$ or (b) $i_{\rho}=F_{\rho}\left(v_{\rho}\right)$ where $F_{\rho}$ is some smooth function from $\mathbf{R}$ to $\mathbf{R}$. Then $\Gamma$ is regularizable.

Proof. The proof proceeds by induction on the number of resistance branches in the given circuit. Suppose the theorem is true for all circuits with $k$ resistance branches. Assume $\Gamma$ has $k+1$ resistance branches and satisfies the hypothesis of the theorem. We will show $\Gamma$ is regularizable. We first pick any resistance branch $R$ in $\Gamma$. $\Gamma$ can be written as

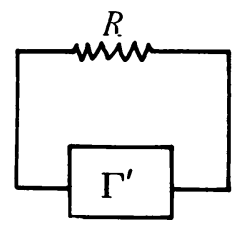

where $\Gamma^{\prime}$ is a circuit with $k$ resistance branches. Thus the circuit $\Gamma_{1}$ defined below can be regularized.

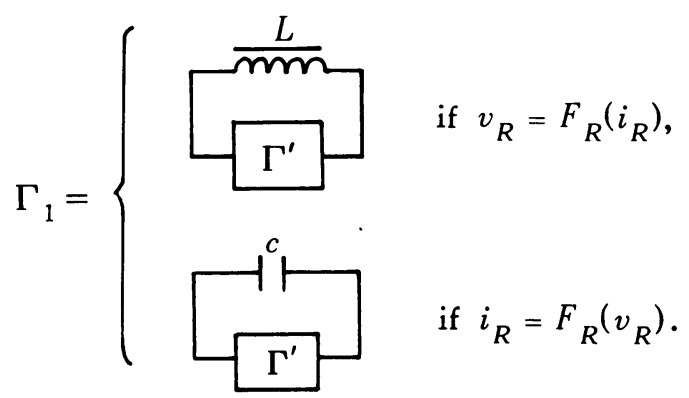

Let us say $\Gamma_{1}$ is regularized by $\Gamma_{2}$ and $\Gamma_{2}^{\prime}$ is $\Gamma_{2}$ without the inductor or capacitor added to $\Gamma^{\prime}$ above. We now claim the circuit 
(a)

(b)

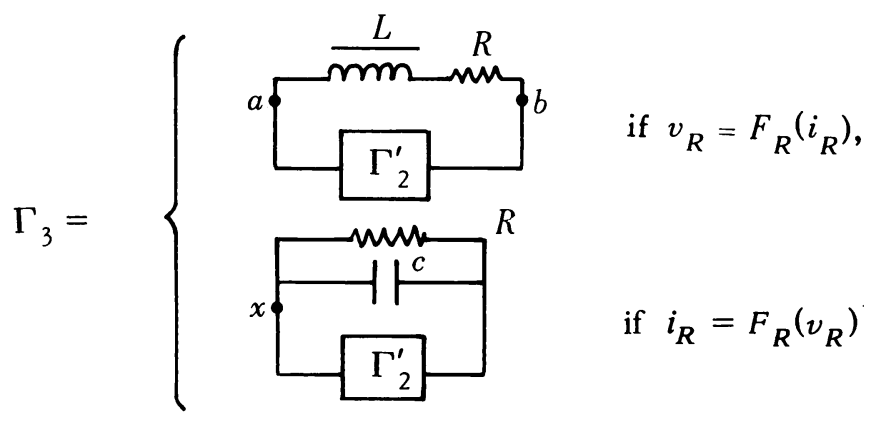

is regular. If we show this, then we are finished since $\Gamma_{3}$ will be a regularization for $\Gamma$. Now since $\Gamma_{2}$ is regular, $\pi_{2}$ has an inverse which gives all the currents and voltages of the circuit in terms of the inductive currents and capacitive voltages. First we observe that this same function will give all the currents and voltages for the branches of $\Gamma_{3}$ in $\Gamma_{2}^{\prime}$. This is so since only the external branch has been altered from $\Gamma_{2}$. The only variable in this branch is $i_{L}$ (case a) or $v_{c}$ (case b), which gives the current and the voltage, respectively, of this branch. Since $i_{L}$ is still the measure of the current in this branch in $\Gamma_{3}$ (case a) and $v_{c}$ is still the measure of the voltage (case b), we may use $\pi_{2}^{-1}$ as $\pi_{3}^{-1} / \Gamma_{2}^{\prime}$. To finish we must determine only $i_{L(c)}$, $\dot{v}_{L(c)}, i_{R}$ and $v_{R}$ in terms of the capacitance voltages and inductance currents of $\Gamma_{3}$. In case (a) we have

$$
i_{L}=i_{R}, \quad v_{R}=F_{R}\left(i_{L}\right), \quad v_{L}=v_{a b}-F_{R}\left(i_{L}\right) .
$$

$v_{a b}$ is determined by capacitors and inductors in $\Gamma_{3}^{\prime}$ since it can be considered as a voltage between two points inside $\Gamma_{2}^{\prime}$. Similarly in case (b) we have

$$
v_{R}=v_{c}, \quad i_{R}=F_{R}\left(v_{c}\right), \quad i_{c}=i_{x}-F_{R}\left(v_{c}\right)
$$

where $i_{x}$ is again determined by Kirchoff's law applied at a vertex in $\Gamma_{2}^{\prime}$ to which the external branch is attached. Thus the inverse is defined and is smooth so that $\pi_{3}$ is a diffeomorphism as desired.

Now to finish our proof we must treat the special case of $k=0$. We must regularize a circuit with no resistances. We define the regularization of $\Gamma$, and call it $\Gamma_{1}$, as follows: We pick a fixed vertex in $\Gamma$, say $x$, and attach a capacitor from $x$ to every other vertex in $\Gamma$. Then we replace every capacitance branch in $\Gamma$ by

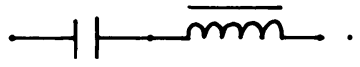

This new circuit is $\Gamma_{1}$. We must show only that it is regular. $\pi_{1}$ in this case is a linear transformation between two vector spaces of the 
same dimension. Thus we need show only that $\operatorname{ker}\left(\pi_{1}\right)=0$. Suppose the voltage across every capacitor is zero. Then the voltage between any vertices must be zero since there is a chain of capacitors connecting them. If the currents in all the inductors are zero then the current in all the branches or altered branches of $\Gamma$ is zero since each contains an inductor. Every remaining branch is a capacitor branch which is connected at one vertex to nothing but branches of the first kind. Thus these branches also have no current by Kirchoff's law, and we are done.

In fact this proof gives us more than just an existence theorem. Using it we can actually construct a regularization of a circuit as seen in the following corollary.

2.4. Corollary. Suppose for each resistance of a circuit $\Gamma$ we have either (a) $v_{\rho}=F_{\rho}\left(i_{\rho}\right)$ or (b) $i_{\rho}=F_{\rho}\left(v_{\rho}\right)$ where $F_{\rho}$ is some smooth function from $\mathbf{R}$ to $\mathbf{R}$. Construct the circuit $\Gamma^{\prime}$ from $\Gamma$ as follows:

(i) Pick a fixed vertex $x$ and connect a capacitor between it and each other vertex in $\Gamma$.

(ii) Every branch in $\Gamma$ should be changed as follows: Replace

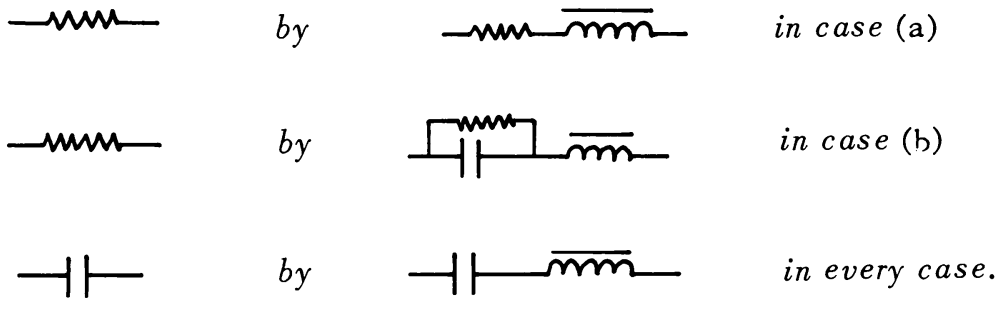

Then $\Gamma^{\prime}$ will be regular.

Proof. Take $\Gamma$ and replace each resistor by either a capacitor or inductor depending on whether the resistor is in case (a) or case (b). Perform step (i) and the last part in step (ii) to get. $\Gamma^{\prime \prime} . \Gamma^{\prime \prime}$ is regular by the theorem. Now add the resistors one by one. The same proof that was used in the induction part of the theorem says that at each addition regularity will be preserved. To show the necessity of conditions (a) or (b) of 2.3 , we give the following example:

2.5 Example. Let $\Gamma$ be the circuit defined below:
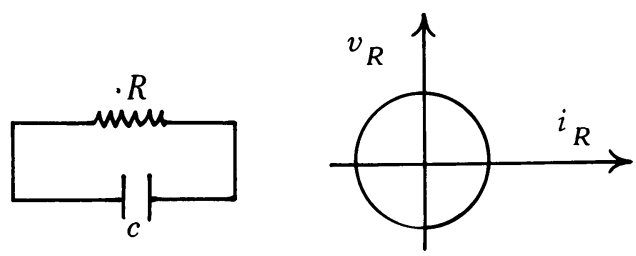
with $i_{R}^{2}+v_{R}^{2}=1$. In this case, if we add $n$ capacitors and inductors we will have $\Sigma \simeq S^{1} \times \mathbf{R}^{n}$ and $\mathcal{Q} \times \mathcal{C}^{\prime}$ will be $\mathbf{R}^{n+1}$. Thus $\pi$ cannot be a diffeomorphism.

We do, however, have the following local result.

2.6. Theorem. Given any circuit and specific values for $v_{\rho}$ and $i_{\rho}$ for all $\rho$, there is a circuit which consists of the original circuit plus capacitors and resistors which is regular so long as $v_{\rho}$ and $i_{\rho}$ remain in a given neighborhood of the given values.

Proof. This theorem is a direct consequence of our original theorem since locally for each resistor either $i_{\rho}=F_{\rho}\left(v_{\rho}\right)$ or $v_{\rho}=F_{\rho}\left(i_{\rho}\right)$.

If our construction were not different for the two different cases 2.3 (a) and (b) then 2.6 would have said that $\pi$ could always be made a local diffeomorphism. As it is, the question of when $\pi$ can be made into a local diffeomorphism by addition of capacitors and conductors is left open.

\section{REFERENCE}

1. S. Smale, On the mathematical foundations of electrical circuit theory, J. Differential Geometry 7 (1972), 193-210.

DEPARTMENT OF MATHEMATICS, UNIVERSITY OF TORONTO, TORONTO, ONTARIO, CANADA

Current address: Department of Mathematics, University of New Brunswick, Fredericton, New Brunswick, Canada 